\title{
Larval midgut modifications associated with Bti resistance in the yellow fever mosquito using proteomic and transcriptomic approaches
}

Guillaume Tetreau $^{1 *}{ }^{*}$, Krishnareddy Bayyareddy ${ }^{2+}$, Christopher M Jones ${ }^{3}$, Renaud Stalinski ${ }^{1}$, Muhammad A Riaz ${ }^{1}$, Margot Paris ${ }^{1}$, Jean-Philippe David', Michael J Adang ${ }^{2,4}$ and Laurence Després ${ }^{1}$

\begin{abstract}
Background: Bacillus thuringiensis var. israelensis (Bti) is a natural larval mosquito pathogen producing pore-forming toxins targeting the midgut of Diptera larvae. It is used worldwide for mosquito control. Resistance mechanisms of an Aedes aegypti laboratory strain selected for 30 generations with field-collected leaf litter containing Bti toxins were investigated in larval midguts at two levels: 1. gene transcription using DNA microarray and RT-qPCR and 2. differential expression of brush border membrane proteins using DIGE (Differential In Gel Electrophoresis).

Results: Several Bti Cry toxin receptors including alkaline phosphatases and $\mathrm{N}$-aminopeptidases and toxin-binding V-ATPases exhibited altered expression levels in the resistant strain. The under-expression of putative Bti-receptors is consistent with Bt-resistance mechanisms previously described in Lepidoptera. Four soluble metalloproteinases were found under-transcribed together with a drastic decrease of metalloproteinases activity in the resistant strain, suggesting a role in resistance by decreasing the amount of activated Cry toxins in the larval midgut.

Conclusions: By combining transcriptomic and proteomic approaches, we detected expression changes at nearly each step of the ingestion-to-infection process, providing a short list of genes and proteins potentially involved in Bti-resistance whose implication needs to be validated. Collectively, these results open the way to further functional analyses to better characterize Bti-resistance mechanisms in mosquitoes.
\end{abstract}

Keywords: Aedes aegypti, Bacillus thuringiensis israelensis, DIGE, Microarray, RT-qPCR, Resistance, Transcriptomics, Proteomics, Midgut, Mosquito, Candidate genes

\section{Background}

Mosquito control represents a major public health concern as mosquitoes transmit many pathogens causing fatal human diseases including malaria, filariasis, dengue, yellow fever, and Chikungunya [1]. Vector borne diseases represent a major health threat and economic burden in disease-endemic countries and are currently expanding worldwide $[2,3]$. As no specific treatment exists for most of these diseases, the most effective way of reducing the incidence of these diseases is to control the vector mosquitoes [4,5]. Chemical insecticides still used in endemic countries have shown their limits as resistance has

\footnotetext{
* Correspondence: guillaume.tetreau@gmail.com

${ }^{\dagger}$ Equal contributors

'Laboratoire d'Ecologie Alpine, LECA-UMR 5553, Université de Grenoble 1, BP 53, 38041, Grenoble cedex 09, France

Full list of author information is available at the end of the article
}

evolved in all target species together with environmental concerns due to their high persistence and toxicity for non-target organisms, including humans [6].

The bacterium Bacillus thuringiensis var. israelensis (Bti) produces a mosquitocidal toxic crystal during sporulation and represents the best alternative to chemical insecticides for mosquito larval control due to its high potency and specificity [7]. The action of Bti begins when larvae ingest $B t i$ spores and toxic crystals. In susceptible larvae, the toxic crystal is dissolved in the alkaline $\mathrm{pH}$ of the midgut, protoxins are then activated by digestive proteases to activated-toxins that bind to specific membrane receptors, form pores, disrupt the midgut epithelium, allowing spore penetration and bacterial proliferation in the host tissues $[7,8]$. The receptors for mosquitocidal Bti Cry toxins are similar to the

\section{Biomed Central}


lepidopteran-active Cry toxins which utilize $\mathrm{N}$ aminopeptidase, alkaline phosphatase and cadherin proteins as midgut receptors [9].

In contrast to Bacillus thuringiensis subspecies active against lepidopteran and coleopteran species where cases of insect resistance in the field have been reported [10-13], only one study reported Bti resistance in field mosquitoes [14]. However, subsequent confirmations of this case have not been reported. The delay in the evolution of resistance to Bti is believed to be due to its composite toxic crystal containing four major toxins (Cry4Aa, Cry4Ba, Cry11Aa and Cyt1Aa) [7]. Cyt toxins are known to largely enhance Cry toxins activity due to synergic effects and to drastically decrease resistance development $[8,15]$. Although $B t i$ is known to have a low persistence in the environment, recent studies suggest that it can persist and possibly proliferate in specific conditions [16-18]. In the French Rhône-Alpes region, decaying leaf litters collected in mosquito breeding sites several months after a Bti treatment revealed a high toxicity against mosquito larvae due to the presence of large amounts of Bti [16]. This toxic leaf litter was used to select an Aedes aegypti strain in laboratory conditions. After 18 generations, the selected strain (named LiTOX) was only moderately resistant to the whole Bti toxins mixture, but up to 30 fold resistant to individual Cry toxins [19]. Although resistance to Bti has already been selected in laboratory conditions [20,21], this is the only reported case of resistance obtained by using fieldcollected material containing residual Bti toxins. Therefore, this Bti-resistant LiTOX strain provides a unique opportunity to better understand the mode of action of $B t i$ toxins and to elucidate the mechanisms of resistance developed by mosquitoes exposed to field residual Bti toxins.

To identify the resistance mechanisms developed by the LiTOX strain, a genome scan and a transcriptome scan were previously performed on whole larvae twelve generations ago [22,23]. The main bias of these wholelarvae approaches is that many genes are identified that may not be directly related to Bti resistance. Indeed, selection was shown to have induced many changes in the LiTOX strain, including decreased egg survival to desiccation, longer larval development time and decreased female fecundity [24], reflecting the evolution of resistance costs that are not directly involved in resistance to Bti toxins. Because insect midgut is the primary target site for Bti toxins our aim in the present work is to focus on constitutive expression changes in midgut proteins of resistant versus susceptible larvae. For that purpose, we combine a comparative analysis of brush border membrane proteins using 2D-DIGE (2-Dimensional Differential in Gel Electrophoresis) with a midgut transcriptome profiling using DNA microarrays. In addition, altered gene expression of known Bti Cry toxins receptors (i.e. alkaline phosphatases, cadherins, N-aminopeptidases) between the two strains were investigated using RTqPCR. Finally, because the DiGE didn't allow detecting proteins with high molecular size such as cadherins, we performed Western blots with anti-cadherins antibodies.

\section{Results}

Resistance levels to Bti toxins in the LiTOX strain

After 30 generations of selection with leaf litter containing $B t i$, bioassays indicated that the LiTOX strain exhibited a moderate 3.5 -fold resistance to commercial Bti mixture Vectobac ${ }^{\circledR}$ WG compared to the susceptible strain at the larval stage (Table 1). When Bti Cry toxins were tested separately, the LiTOX strain showed an increased resistance of 68-fold, 9-fold and 9-fold to Cry4Aa, Cry4Ba and Cry11Aa protoxins respectively. The relatively important variability observed for the $\mathrm{LC}_{50}$ for Cry4Aa toxin of the LiTOX strain is mainly due to a higher variability in larval mortality in the replicates than for the susceptible strain and for the other toxins. As resistance is not fixed yet in the LiTOX strain [24], this variability between replicates might reflect a large range of different combinations of Cry4A resistance alleles between individuals.

\section{Midgut transcriptome profiling}

Comparative transcriptome profiling between total mRNAs extracted from midguts of larvae from the LiTOX and the susceptible strains was performed using a DNA microarray representing 14204 of the more than 17000 Ae. aegypti transcripts identified in Vectorbase. A total of 3512 transcripts were detected in at least 5 hybridizations out of 6 [ArrayExpress: E-MTAB-1094] (Additional file 1). Among them, 24 and 46 genes were significantly over- and under-transcribed respectively in the LiTOX strain $(\geq 3$-fold and corrected P-value $<0.01$ )

Table 1 Lethal concentrations and resistance ratio for the LiTOX and susceptible strains for Bti and Cry toxins

\begin{tabular}{llll}
\hline Toxins & Strain & $\mathbf{L C}_{\mathbf{5 0}}$ in $\mathbf{n g} / \mathbf{m L}(\mathbf{9 5} \% \mathbf{C l})$ & $\mathbf{R R}_{\mathbf{5 0}}$ \\
\hline Cry4Aa & Susceptible & $646.28(514.20-826.93)$ & $/$ \\
& LiTOX & $43873.12(28396.11-78207.31)$ & $\mathbf{6 7 . 9}$ fold \\
Cry4Ba & Susceptible & $322.27(228.39-468.57)$ & $/$ \\
& LiTOX & $2922.26(1924.95-4168.76)$ & $\mathbf{9 . 1}$ fold \\
Cry11Aa & Susceptible & $156.14(112.86-219.46)$ & $/$ \\
& LiTOX & $1434.81(1146.52-1774.22)$ & $\mathbf{9 . 2}$ fold \\
Bti Vectobac WG & Susceptible & $90.6(79.89-101.12)$ & $/$ \\
& LiTOX & $312.6(277.27-359.49)$ & $\mathbf{3 . 5}$ fold \\
\hline
\end{tabular}

Lethal concentrations $\mathbf{5 0 \%}\left(\mathrm{LC}_{50}\right)$ of the resistant (LiTOX) and the susceptible strain for the three Cry toxins (Cry4Aa, Cry4Ba and Cry11 Aa) and for the commercial Bti at $\mathbf{2 4} \mathbf{h}$. Resistance ratios $\mathbf{5 0}\left(\mathbf{R R}_{\mathbf{5 0}}\right)$ are calculated for each product as $\mathrm{LC}_{50}$ of LiTOX divided by $\mathrm{LC}_{50}$ of BoraBora strain. $L C_{50}$ are expressed in $\mathrm{ng} / \mathrm{mL}$. 
(Additional file 2). Distribution of transcription ratios was well balanced between over and under-transcription ranging from 20.9-fold under-expression to 18.9-fold over-expression (Figure 1). RT-qPCR validation of transcription ratios for 15 selected genes revealed a good correspondence between the two techniques, supporting the reliability of microarray data (Additional file 3).

Differentially transcribed genes were further analyzed according to their biological function by classifying them into 13 different categories (Figure 2). Genes of unknown function represented $34 \%$ of detected transcripts while genes not assigned to any category (other functions) represented 17\%. Enzymes represented 30\% of detected transcripts and were strongly over-represented among under- and over-transcribed genes (55\% and 60\% respectively) (Figure $2 \mathrm{~B} \& \mathrm{C}$ ). Proteases were equally represented in over- and under-expressed genes, while detoxification enzymes were more often under- than over-transcribed (7 under-transcribed versus 2 overtranscribed genes). Transaminases, represented by only 11 genes in the Ae. aegypti genome, were overrepresented in under- and over-transcribed genes while dehydrogenases were strongly over-represented only in over-transcribed genes (23\% of enzymes compared to $10 \%$ overall).

\section{Midgut differential proteomics}

Midgut membrane proteins were compared between larvae of the LiTOX and susceptible strains using 2DDIGE (Figure 3). Dye-swapping for each biological sample showed no dye-dependent spot changes on the gels (Additional file 4). Spot locations were reproducible between the biological replicates, but the signal intensity was higher for the second replicate, revealing additional spots differing between the two strains (Additional file 4). A total of 56 distinct protein spots differently expressed between the two strains were processed and 35 unique proteins were identified (Figure 3B, Additional file 5). The MS/MS analyses gave the same protein identifications between biological replicates for spots 2, 8, 20, 21 and 24 with high Mascot scores (from 110 to 249) while spots 14, 42 and 49, showing Mascot scores lower than 100, were assigned to different proteins (Additional file 5). Indeed, none of the spots with low Mascot scores were considered for further analyses. Different spots yielded the same identified protein for 10 proteins, with a maximum of six spots for AAEL005798 (V-ATP synthase subunit beta).

\section{Genes and proteins differentially expressed in the LiTOX} strain

Proteome analysis identified two $\mathrm{N}$-aminopeptidase proteins (APN, annotated as 'protease $\mathrm{m} 1$ zinc metalloprotease') differentially expressed in the LiTOX strain (Table 2): two spots matching APN AAEL012774 were upregulated and two of the three spots matching APN AAEL012776 were down-regulated in the LiTOX strain. Transcriptomic approach detected thirteen APN (including AAEL012774 and AAEL012776) with transcription level ranging from -1.82 to +1.96 fold changes (Additional file 1 ) but none was significant.

Two alkaline phosphatases (ALP) proteins, matching AAEL003313 and AAEL003298, were under-expressed in the LiTOX strain while transcriptomics identified two

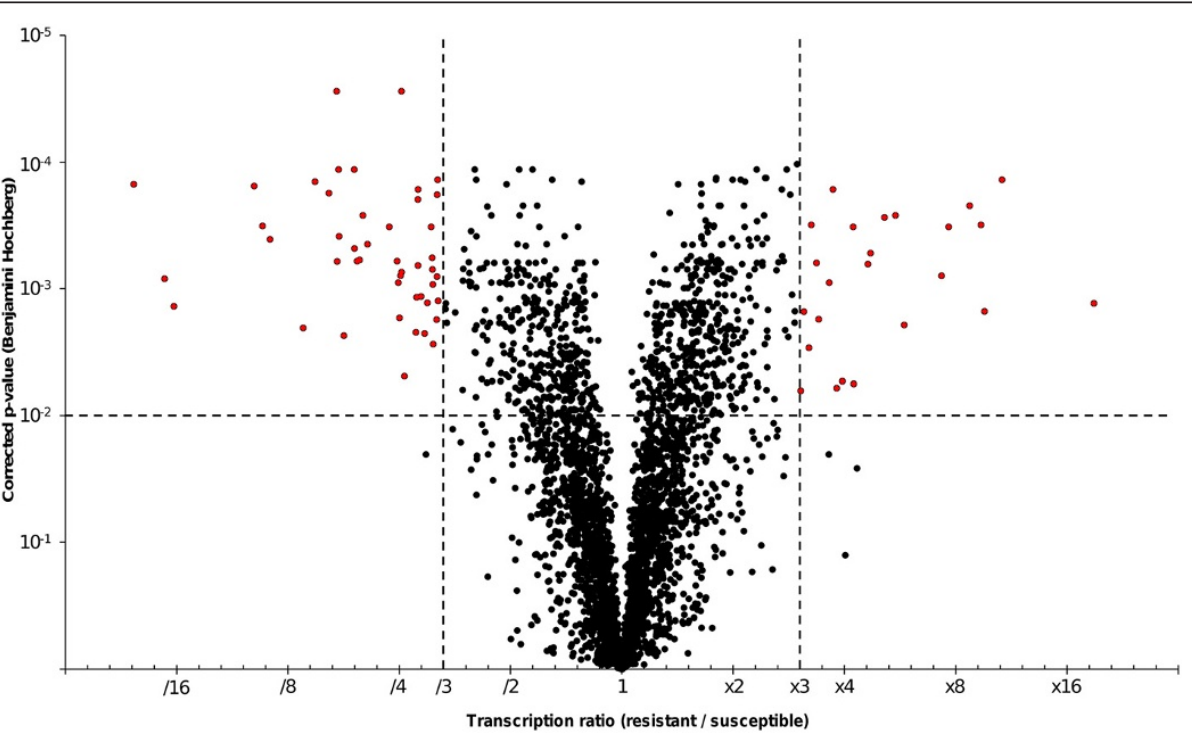

Figure 1 Volcano plot of differentially-transcribed genes identified by microarray analysis. The Benjamini-Hochberg P-values were plotted against the fold change in gene expression for all genes. The horizontal lines in the plot represent the statistical test significance 0.01 and the vertical bars represent the genes at least three-fold up- or down-regulated in LiTOX Bti-resistant strain compared to Bora-Bora susceptible strain. 



Figure 2 Functional analyses of all the genes detected and genes differentially expressed in the resistant strain. Circle charts of the biological functions of all the genes detected $(\mathbf{A})$, those under-expressed $(\mathbf{B})$ and over-expressed $(\mathbf{C})$ in the LiTOX strain compared to the susceptible Bora-Bora strain. Genes are classified into 13 categories: receptors (orange), transport (green), DNA interaction (purple), cytoskeleton (dark blue), ribosomal proteins (light blue), proteases (black), detoxication enzymes (brown), kinases/phosphatases (orange), transaminases (red), dehydrogenases (dark pink), other enzymes (pink), other functions (dark grey) and unknown functions (light grey).

other ALP genes (AAEL011175 and AAEL015070) significantly over-transcribed in the LiTOX strain with transcription ratios of +4.63 and +3.95 fold respectively. All ALPs but AAEL003298 have predicted glycosylphosphatidylinositol (GPI)-anchor domains allowing them to tether to the epithelial membrane and be potential membrane-bound Cry toxin receptors (Additional file 6).

Four proteins matching ATP synthase subunits alpha, beta and epsilon, with two to seven different spots for the same protein, had levels from -1.51 to

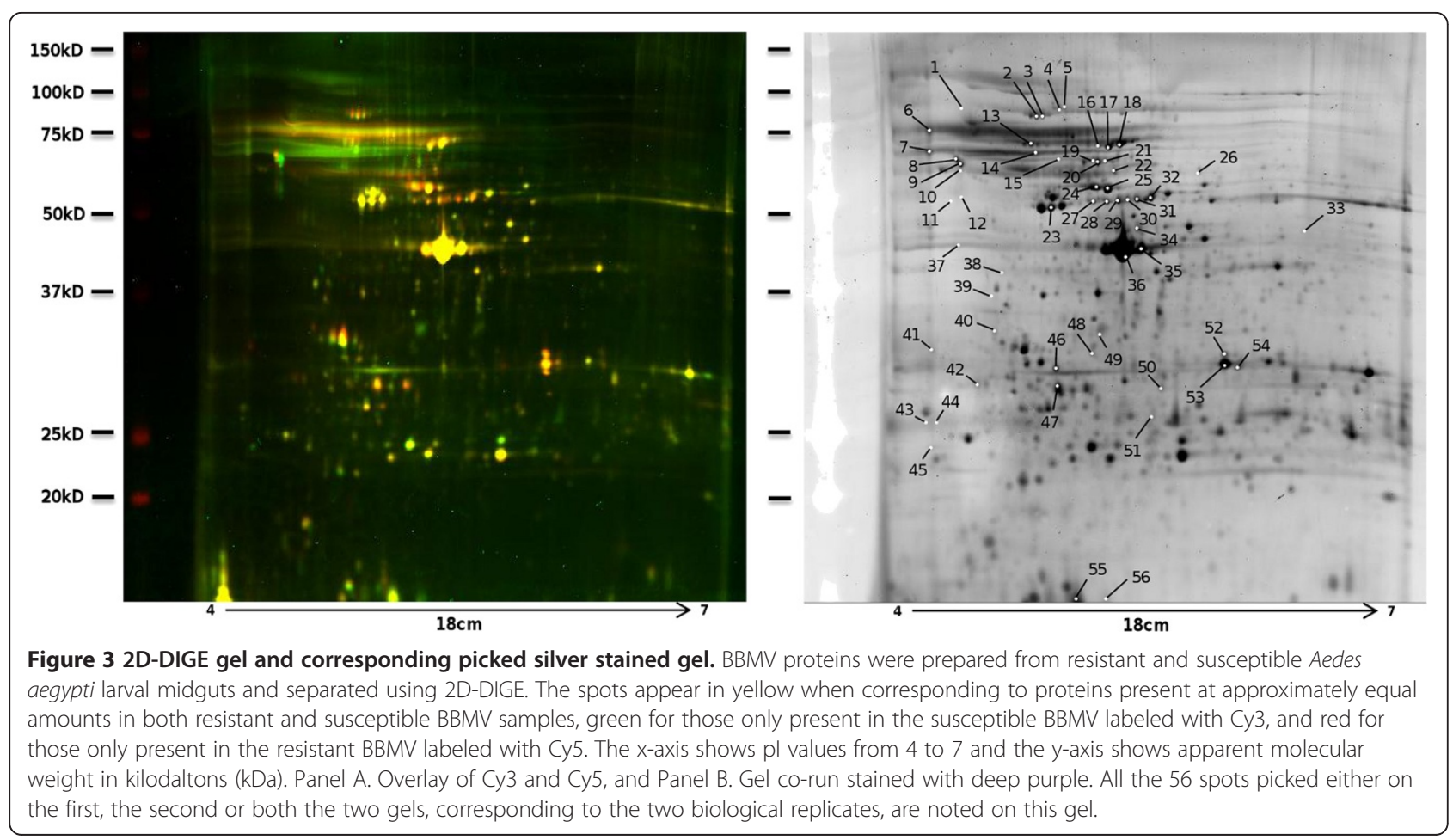


Table 2 Protein identification of 30 spots with highest Mascot scores picked on deep purple stained 2D-gel

\begin{tabular}{|c|c|c|c|c|c|c|c|c|c|}
\hline \multirow{2}{*}{\multicolumn{2}{|c|}{$\begin{array}{ll}\text { Spot } & \mathbf{F} \\
\mathbf{N b} & \mathbf{c} \\
\text { Proteases }\end{array}$}} & $\begin{array}{l}\text { Fold } \\
\text { changes }\end{array}$ & \multirow[t]{2}{*}{$\begin{array}{l}\text { Vectorbase } \\
\text { access number }\end{array}$} & \multirow[t]{2}{*}{$\begin{array}{l}\text { Mascot } \\
\text { score }\end{array}$} & \multirow[t]{2}{*}{ Top ranking match } & \multirow[t]{2}{*}{ Predicted PI } & \multirow[t]{2}{*}{$\begin{array}{l}\text { Predicted } \\
\text { mass }(\mathrm{kDa})\end{array}$} & \multirow[t]{2}{*}{$\begin{array}{l}\text { \% sequence } \\
\text { coverage }\end{array}$} & \multirow[t]{2}{*}{ Species } \\
\hline & & & & & & & & & \\
\hline 2 & - & 1.88 & AAEL015386_a & 249 & dipeptidyl-peptidase & 4.91 & 84.9 & 42 & Ae. aegypti \\
\hline 3 & - & 2.77 & AAEL015386_b & 149 & dipeptidyl-peptidase & 4.91 & 84.9 & 22 & Ae. aegypti \\
\hline 4 & + & 1.81 & AAEL012774_a & 237 & protease $\mathrm{m} 1$ zinc metalloprotease & 4.81 & 102.5 & 41 & Ae. aegypti \\
\hline 5 & + & 2.34 & AAEL012774_b & 162 & protease $\mathrm{m} 1$ zinc metalloprotease & 4.81 & 86.7 & 42 & Ae. aegypti \\
\hline 20 & + & 1.81 & AAEL012776_a & 135 & protease $\mathrm{m} 1$ zinc metalloprotease & 5.19 & 103.3 & 30 & Ae. aegypti \\
\hline 21 & - & 2.74 & AAEL012776_b & 146 & protease $\mathrm{m} 1$ zinc metalloprotease & 5.19 & 103.3 & 27 & Ae. aegypti \\
\hline 19 & - & 3.81 & AAEL012776_c & 136 & protease $\mathrm{m} 1$ zinc metalloprotease & 5.19 & 103.3 & 22 & Ae. aegypti \\
\hline \multicolumn{10}{|c|}{ Detoxification enzymes } \\
\hline 45 & + & 3.01 & CPIJ019700 & 119 & cytochrome P450 & 7.6 & 58.4 & 41 & C. quinquefasciatus \\
\hline \multicolumn{10}{|c|}{ Kinases-Phosphatases } \\
\hline 22 & - & 1.58 & AAEL003313 & 62 & alkaline phosphatase & 5.46 & 61.0 & 23 & Ae. aegypti \\
\hline 24 & - & 1.92 & AAEL003298_a & 186 & alkaline phosphatase & 5.28 & 58.8 & 39 & Ae. aegypti \\
\hline 25 & - & 1.16 & AAEL003298_b & 194 & alkaline phosphatase & 5.23 & 58.3 & 39 & Ae. aegypti \\
\hline \multicolumn{10}{|c|}{ Other enzymes } \\
\hline 6 & + & 1.78 & AAEL010532 & 146 & alpha-amylase & 4.82 & 68.9 & 37 & Ae. aegypti \\
\hline 13 & - & 1.27 & AAEL004580 & 129 & beta-galactosidase & 4.87 & 74.1 & 34 & Ae. aegypti \\
\hline 11 & - & 2.06 & AAEL002827_a & 61 & ATP synthase beta subunit & 5.03 & 53.9 & 32 & Ae. aegypti \\
\hline 23 & - & 2.29 & AAEL002827_b & 152 & ATP synthase beta subunit & 5.03 & 53.9 & 55 & Ae. aegypti \\
\hline 16 & - & 2.06 & AAEL008787_a & 162 & V-ATP synthase subunit alpha & 5.26 & 68.5 & 31 & Ae. aegypti \\
\hline 17 & - & 2.19 & AAEL008787_b & 231 & V-ATP synthase subunit alpha & 5.26 & 68.5 & 42 & Ae. aegypti \\
\hline 18 & - & 1.82 & AAEL008787_c & 132 & V-ATP synthase subunit alpha & 5.26 & 68.5 & 30 & Ae. aegypti \\
\hline 27 & - & 1.51 & AAEL005798_a & 200 & V-ATP synthase subunit beta & 5.31 & 54.8 & 49 & Ae. aegypti \\
\hline 28 & - & 1.69 & AAEL005798_b & 177 & V-ATP synthase subunit beta & 5.31 & 54.8 & 44 & Ae. aegypti \\
\hline 29 & - & 1.72 & AAEL005798_c & 187 & V-ATP synthase subunit beta & 5.31 & 55.4 & 53 & Ae. aegypti \\
\hline 30 & - & 2.06 & AAEL005798_d & 229 & V-ATP synthase subunit beta & 5.38 & 55.5 & 52 & Ae. aegypti \\
\hline 31 & - & 2.39 & AAEL005798_e & 197 & V-ATP synthase subunit beta & 5.38 & 55.4 & 48 & Ae. aegypti \\
\hline 32 & - & 1.89 & AAEL005798_f & 181 & V-ATP synthase subunit beta & 5.38 & 55.4 & 56 & Ae. aegypti \\
\hline 52 & - & 2.14 & AAEL012035_a & 93 & V-ATP synthase subunit E & 5.91 & 25.7 & 35 & Ae. aegypti \\
\hline 53 & - & 2.21 & AAEL012035_b & 88 & V-ATP synthase subunit E & 5.91 & 25.7 & 38 & Ae. aegypti \\
\hline \multicolumn{10}{|c|}{ Other functions } \\
\hline 8 & + & 10.74 & AAEL001005_a & 195 & calreticulin & 4.42 & 47.0 & 43 & Ae. aegypti \\
\hline 8 & - & 2.56 & AAEL001005_a & 226 & calreticulin & 4.42 & 47.0 & 49 & Ae. aegypti \\
\hline 9 & + & 3.14 & AAEL001005_b & 210 & calreticulin & 4.42 & 47.0 & 49 & Ae. aegypti \\
\hline 10 & + & 2.75 & AAEL001005_c & 158 & calreticulin & 4.42 & 47.0 & 48 & Ae. aegypti \\
\hline 12 & + & 3.01 & AAEL001005_d & 93 & calreticulin & 4.42 & 46.7 & 35 & Ae. aegypti \\
\hline
\end{tabular}

Proteins are classified according to their putative function using the same 13 categories as for transcriptomic data. When different spots pointed to the same protein, they were differentiated using letters after the access number. For each identification, the predicted pl, the predicted mass in kiloDaltons $(\mathrm{kDa})$, the percentage of sequence coverage and the species and database matched are indicated.

-2.39 lower in the LiTOX strain. This tendency was consistent with microarray data for AAEL008787-RA $(-1.19$ fold $), \quad$ AAEL005798 $(-1.37$ fold $)$ and AAEL012035 (-1.19 fold) although P values were not significant.
A unique calreticulin protein was picked and identified from DIGE gels. Initially detected as only one big spot with +10.74 fold change, the second biological replicate allowed clearly identifying four different spots respectively $-2.56,+2.75,+3.01$ and +3.14 fold 
differentially expressed in the LiTOX strain. In microarray experiment, no significant differential transcription of this gene was found in the LiTOX strain.

Using BLASTP software, we managed to identify putative functions for the 15 transcripts of unknown function differentially transcribed in the LiTOX strain with protein identities ranging from 25 to $99 \%$ (Additional file 2). Among them, two were strongly over-transcribed in the resistant strain (AAEL013584 19-fold and AAEL010435 9.6-fold) and matched to a putative $\mathrm{G}_{12}$ protein in $A e$. aegypti (77\% protein identity, Additional file 2).

Among the five cytochrome P450 monooxygenase transcripts identified by microarray analyses, CYP4D24 was over-transcribed while the others (CYP6N9, CYP6Z7, CYP6Z8 and CYP9M9) were under-transcribed in the LiTOX strain. DIGE experiments identified one protein matching to a cytochrome P450 3-fold overexpressed in the LiTOX strain.

Transcriptomic data detected four metalloproteinases significantly under-transcribed from -3.16 to -5.29 fold (Additional file 2). The presence of conserved domains of soluble astacin-like metalloproteinases together with the absence of detected GPIanchor domain (Additional file 6) suggests that these four metalloproteinases are probably secreted extracellular enzymes, explaining why they were not identified in the BBMV by the DIGE analysis.

\section{Global and specific proteolytic activities}

To determine if the modifications in protease transcription levels observed in the resistant strain result in changes in gut proteolytic activities, we compared the protease activities of secreted proteins from larval midgut of each strain using azocasein as substrate. Total proteolytic activity was $8.5 \%$ higher in the resistant strain compared to the susceptible strain (Table 3 ). The use of specific protease inhibitors revealed that more than $90 \%$ of the total proteolytic activity is due to serine protease for both strains. Among them, chymotrypsins and trypsins represented respectively more than $40 \%$ and $20 \%$ of the total activity in both strains. The use of the metalloproteinase inhibitor EDTA showed that $12 \%$ of the total proteolytic activity was due to metalloproteinases in the susceptible strain whereas no inhibition was measured in the resistant strain (Wilcoxon test; P-value
$<0.05)$, suggesting a strong reduction of metalloproteinase activity in the LiTOX strain.

\section{Discussion}

Resistance levels to Bti toxins in the LiTOX strain

After 30 generations of selection, resistance to Cry4Aa in the LiTOX strain has more than doubled as compared to twelve generations ago, while resistance ratios did not change for Cry4Ba and Cry11Aa [19,22]. Resistance to Bti is moderate (3.5 fold) but higher than at generation 18 (2-fold), indicating that resistance alleles are not all fixed yet. These results are consistent with previous attempts to select Ae. aegypti, Culex pipiens and $C x$. quinquefasciatus with Bti which obtained moderate resistance ( 2 to 3 fold) after 20 to 30 generations $[20,21,25,26]$. The increased Bti resistance observed may be due to the increase in Cry4Aa resistance, and most changes observed in the present study may be related to Cry4Aa resistance. The discrepancy between Bti and Cry4Aa increased resistances is likely to be due to the presence of Cyt toxin in Bti, known to overcome Cry resistance in insects [15].

\section{Midgut transcriptome and proteome analyses}

Our comparison of midgut transcripts and brush border proteins between the susceptible and LiTOX strains revealed an overlapping but distinct set of transcripts/proteins differentially expressed. Transcriptome profiling with a microarray representing more than $81 \%$ of known $A e$. aegypti transcripts lead to the detection of 3512 transcripts of which 70 were differentially transcribed in the LiTOX strain. This relatively low number of transcripts detected (about 25\%) is probably due to the low transcription level or absence of transcription of several genes in this particular organ - the larval midgut. Little overlap was observed between the previous transcriptomic analysis, performed on whole larvae 12 generations ago using a DGETP approach [23], and the present study, focusing on midgut gene expression using microarrays. This is possibly due to the technical differences between the two studies and to the fact that no resistance gene is fixed yet in the LiTOX strain, indicated by the still increasing resistance to Bti and to Cry toxins [19]. Moreover, as genes conferring resistance to Bti toxins are likely to be expressed in larval midgut, focusing on midguts rather

Table 3 Total enzymatic activity and effect of protease inhibitors on the azocaseinolytic activity of midgut extract

\begin{tabular}{|c|c|c|c|c|c|}
\hline \multirow[t]{2}{*}{ Strain } & \multirow[t]{2}{*}{ Total enzymatic activity (OD at $440 \mathrm{~nm}$ ) } & \multicolumn{4}{|c|}{ Percentage of inhibition of total activity (\%) } \\
\hline & & PMSF & TLCK & TPCK & EDTA \\
\hline Susceptible & $0.328 \pm 0.009$ & $92.98 \pm 0.57$ & $46.82 \pm 1.02$ & $23.42 \pm 2.24$ & $12.18 \pm 1.74$ \\
\hline LiTOX & $0.356 \pm 0.010$ & $92.31 \pm 0.23$ & $42.01 \pm 2.29$ & $20.87 \pm 1.64$ & $-1.74 \pm 4.55$ \\
\hline Wilcoxon test & $*$ & NS & NS & NS & $*$ \\
\hline
\end{tabular}


than on whole larvae allows to considerably reduce the candidate gene dataset, and to consider only genes likely to be directly involved in resistance, rather than those only indirectly affected by selection side-effects (genetic drift) or compensatory mechanisms (resistance costs) [24]. The 2D-DIGE analysis resolved about 400 distinct proteins in larval BBMV fractions, 56 spots were picked of which 50 differed by more than 2 -fold between the two strains. The difference between the number of spots picked (56) and the number of unique identified proteins (35) is due to different spots for the same protein, as for example up to six spots observed for one V-ATPase. The multiple spots for the same protein are most likely due to post-translational modifications (glycosylation, phosphorylation) that cause shift in protein mobility. Eight spots common to the replicated DIGE experiments were picked and identified twice. Among them all but one, calreticulin (AAEL01005), showed similar levels of differential expression supporting the consistency of biological replicates (Additional file 4). Both transcriptomic and proteomic data identified more under than over-expressed genes/proteins in the LiTOX strain, which is congruent with a previous transcriptome analysis performed on whole larvae 12 generations ago [23]. Such asymmetry is not surprising considering that mechanisms of resistance to $B t$ can involve a decreased activation of protoxins or a decreased toxin-binding to the epithelium membrane [27].

Little overlap was found between data obtained by transcriptomic and proteomic approaches. This could be explained by both biological processes and technical limitations inherent to each method. Regarding DIGE, BBMV were used, which are enriched for proteins attached to apical brush border midgut membrane via scaffolding and proteins attached to the inner membrane leaflet via acylation. Therefore, except few soluble proteins trapped in re-folded membranes, soluble intracellular proteins and proteins excreted inside the gut lumen are typically absent in BBMV preparations [28,29]. In contrast, mRNAs extracted from whole larval midguts should be representative of all transcripts present in midgut cells. Another factor limiting overlapping data may be the consequence of the relatively stringent filtering of the microarrays dataset (3-fold threshold). Several studies also showed that mRNA transcription profiles fit poorly with protein levels because of numerous posttranscriptional regulatory activities and post-translational events [30-32]. Such events generate a high diversity of proteins while gene expression remains unchanged, and this source of variation is so far under-explored in studies on fast adaptive changes like the evolution of insecticide resistance. It is likely that the two complementary approaches used in the present work detected distinct mechanisms of resistance acting at different steps in the mode of action of Bti (i.e. crystal solubilization, toxin activation and binding to receptors).

\section{Altered expression and activities of proteases from the LiTOX strain}

Four soluble astacin-like metalloproteinases were found significantly under-transcribed in the LiTOX strain. This observation was correlated with a strong decrease of metalloproteinases activity among the enzymes secreted in the midgut lumen of larvae from the resistant strain. To our knowledge, this is the first time astacin-like metalloproteinases are associated to $B t$ resistance. The observed decrease in metalloproteinases in the resistant strain might reflect an alteration in Bti Cry toxins activation in the gut lumen of LiTOX larvae. Further experiments based on measuring proteolytic activities and performing bioassays with activated toxins will clarify the potential role that alteration of protoxins processing, notably for Cry4Aa, could play in the resistance phenotype.

\section{Altered expression of known Bti-binding proteins in the LiTOX strain}

To validate the expression alteration of putative Btireceptors observed in microarrays and DIGE approaches, RT-qPCR analyses were performed on five $\mathrm{N}$-aminopeptidases (APN1 to 5), two cadherins (Cad1 and Cad2) and three alkaline phosphatases (ALP1 to 3) previously described as binding proteins for Cry4Ba [29] or Cry11Aa [33-36] (Table 4).

The cadherin described as a Cry11Aa-receptor in Ae. aegypti (AAEL007488) [34] was found 1.47 fold undertranscribed in both microarrays and RT-qPCR experiments. However, no cadherin was detected by DIGE approach. The inability to detect cadherin in the DIGE analysis is not surprising as they are large proteins $(>170 \mathrm{kDa})$ present in low amounts in insect brush border membranes [37]. Blotting of BBMV using two anti-cadherin antibodies showed that most of the cadherins were degraded, even in a freshly prepared UGAL Aedes BBMV preparation, confirming that cadherins in BBMV are very unstable (Additional file 7). Western blots showed that cadherin(s), notably a $\sim 32 \mathrm{kDa}$ fragment, is strongly over-represented in the LiTOX strain compared to the susceptible strain (Additional file 7). Further analyses of the toxins-binding properties of the detected cadherin(s) are needed to better understand the role they could play in the resistance phenotype.

Alkaline phosphatases (ALPs), typically anchored by GPI-moieties, are known to be Cry toxin receptors in Lepidoptera [38,39] and mosquitoes [36,40]. Recently, a decrease in ALP amounts and activities were linked to Cry-resistance of larvae from three lepidopteran genera [41]. The ALPs detected as over-transcribed in the LiTOX strain by the two transcriptomic approaches were either not identified as differentially expressed (AAEL000931, AAEL009077 and AAEL015070) or identified as under- 
Table 4 Altered expression of known Bti Cry-binding proteins detected by transcriptomic and proteomic approaches

\begin{tabular}{|c|c|c|c|c|c|c|}
\hline Gene & Accession number & Microarrays & RT-qPCR & 2D-DIGE & Binding protein & Ref \\
\hline Alkaline phosphatase (ALP1) & AAEL000931 & ND & +11.40 & $\mathrm{NI}$ & Cry $11 \mathrm{Aa}$ & [33] \\
\hline Alkaline phosphatase (ALP2) & AAEL003298 & -1.57 & -1.56 & $-1.92 ;-1.16$ & Cry4Ba & [29] \\
\hline Alkaline phosphatase (ALP3) & AAEL003313 & +1.15 & +1.52 & -1.58 & Cry4Ba & [29] \\
\hline Alkaline phosphatase (ALP4) & AAEL009077 & ND & +1.15 & $\mathrm{NI}$ & Cry11Aa & [36] \\
\hline Alkaline phosphatase (ALP5) & AAEL015070 & +3.95 & +7.69 & $\mathrm{NI}$ & Cry $4 \mathrm{Ba}$ & [29] \\
\hline Cadherin (Cad1) & AAEL007478 & ND & -1.11 & UD & Cry $11 \mathrm{Aa}$ & [34] \\
\hline Cadherin (Cad2) & AAEL007488 & -1.47 & -1.46 & UD & Cry $11 \mathrm{Aa}$ & [34] \\
\hline N-Aminopeptidase (APN1) & AAEL012774 & +1.44 & +1.26 & $+1.80 ;+2.34$ & Cry $11 \mathrm{Aa}$ & [35] \\
\hline N-Aminopeptidase (APN2) & AAEL012776 & -1.34 & -1.62 & $+1.81 ;-2.74 ;-3.81$ & Cry $4 \mathrm{Ba}$ & [29] \\
\hline N-Aminopeptidase (APN3) & AAEL012778 & -1.04 & +1.20 & $\mathrm{NI}$ & Cry $11 \mathrm{Aa}$ & [35] \\
\hline
\end{tabular}

Given values indicate the level of expression in the LiTOX strain compared to the susceptible strain detected in microarrays, RT-qPCR and DIGE experiments. ND, Non detected in at least 5 of the 6 microarray hydribizations; NI, Non identified as differentially expressed between the two strains and therefore non-picked for MS/MS identification; UD, Undetectable in 2D-DIGE due to their high molecular weight and their low amount in BBMV.

expressed (AAEL003313) by DIGE approach. These results suggest that the lower ALP protein abundance in the epithelium membrane might rather be due to posttranslational events than under-expression. Indeed, our DIGE analyses identified three ALPs showing a decreased expression in the LiTOX strain (AAEL003313 and two spots of AAEL003298). Moreover, the ALP AAEL003298 was also detected as under-transcribed in microarrays, RT-qPCR and in a previous transcriptomic study [23]. These two ALP have already been described as Cry4Babinding proteins [29]. The reduction of potential Cryreceptor ALPs proteins on the brush border of LiTOX larvae is consistent with the resistant phenotype.

$\mathrm{N}$-Aminopeptidases (APNs) are a third major class of Cry toxin receptors in Lepidoptera [42] and mosquitoes $[43,44]$ and their alteration correlates with Cry1Aresistance in Helicoverpa armigera [45] and Trichoplusia ni [46]. DIGE experiments revealed two spots of APN AAEL012776 being under-expressed, congruent with transcriptomic data, while another spot was overexpressed in the LiTOX strain. Two spots matching APN AAEL012774 were over-expressed in the LiTOX strain, as also found by transcriptomic approaches. These two ALP proteins have been previously described as potential receptors for Cry11Aa in Ae aegypti [35] but it is still unclear how their altered expression could lead to a higher Bti-resistance.

In general, $B t$ resistance involves changes in the Cry receptors structure rather than in their expression $[27,47]$, although some cases of differential expression of cadherin and aminopeptidase have been reported in resistant strains [48-50]. These changes in expression can be the result of diverse genetic mechanisms including mutations in regulatory regions or even genome rearrangements that can drive rapid adaptation to new environmental pressures such as an insecticide treatment.
Moreover, in the case of $B t i$, the presence of Cyt toxins, known to act as Cry receptors [51], might contribute to overcome receptor alterations in the LiTOX strain. Further analysis of the binding capacities of Cry toxins to the putative receptors found differentially expressed here will contribute to evaluate their relative roles in Bti resistance. Only few studies have focused on Cry4Aa toxin binding to our knowledge [52,53] and nothing is known about its potential membrane receptors. Such experiments will determine if its receptors are highly specific, explaining the high differences in the resistance ratio between Cry4Aa and the other Cry toxins in the LiTOX strain, or if Cry4Aa shares all or a part of its receptors with Cry4Ba and Cry11Aa that could lead to crossresistance.

\section{Other mechanisms potentially involved in the resistance}

All the spots of the four ATP synthases detected by our proteomic approach showed an under-expression pattern in the LiTOX strain. Vacuolar $\mathrm{H}^{+}$-ATPases (V-ATPase) subunits B to E are known to bind Cry4Ba [29] and subunits $A$ and $B$ have been described to bind for Cry1Ac in Heliothis virescens [54] and Helicoverpa armigera [55]. Moreover, V-ATPases are localized in the posterior midgut of mosquito larvae [56], where Cry4Aa, Cry4Ba and Cry11Aa toxins exhibit the highest affinity to the epithelium membrane $[52,53,57,58]$. Nevertheless, their role as Bti toxins receptors has not been demonstrated yet. VATPases are strongly implicated in the alkalinization of the midgut $\mathrm{pH}$ by establishing a proton motive force by transporting proton across membranes leading to a $\mathrm{pH}$ gradient and transmembrane voltage [59-61]. Onken et al. (2008) inhibited all the proteins implicated in the alkalinization process in the midgut of Ae. aegypti larvae and they showed that only the inhibition of V-ATPases induced a strong acidification of the midgut $\mathrm{pH}$ [62]. As $\mathrm{pH}$ affects 
numerous aspects of toxin action like $B t$ crystal solubility [7], toxin conformation [63,64], gut enzymes activity [65] and pore formation [66,67], an alteration of gut $\mathrm{pH}$ could have a general effect on reducing Bti toxicity. Comparing internal larval midgut $\mathrm{pH}$ between resistant and susceptible strains will allow to confirm/infirm if the observed ATPases decreased expression induce an acidification of the gut lumen.

Multiple detoxification enzymes were found undertranscribed in the resistant strain. Such enzymes are often involved in the degradation of small chemicals such as insecticides and plant allele-chemicals $[68,69]$, but they are unlikely to process large proteins such as Bti toxins. Synthesis of detoxification enzymes represents an important energetic cost for the insect [70]. Moreover, several detoxification genes found under-transcribed in our dataset, were found over-transcribed in Ae. aegypti larvae submitted to a chemical challenge [71]. Although the over-expression of particular detoxification genes in the resistant strain can be linked to larval response to tannins contained in the toxic leaf litter [72], the frequent under-expression of these enzymes in the resistant strain may reflect compensatory mechanisms.

\section{Conclusion}

Bti has evolved to infect Diptera such as mosquitoes and blackflies through a sequential mechanism. The multistep mode of action of Bti and its toxins from ingestion to spore germination and proliferation offers many resistance ways for mosquito larvae. By combining transcriptomic and proteomic approaches, we detected expression alteration at nearly each step of the ingestion-to-infection process. Our study paves the way to further functional studies to characterize resistance mechanisms to this bioinsecticide. This information will be of extreme value as this environmentally safe bioinsecticide is increasingly used for vector control worldwide with virtually no knowledge and no suspicion so far about how mosquitoes can develop resistance in the field.

\section{Methods}

\section{Mosquito strains}

The Ae. aegypti laboratory strain Bora-Bora, susceptible to all insecticides, was used for selection with fieldcollected leaf litter containing Bti spores and toxins [16]. This material, highly toxic after ingestion by mosquito larvae, was used for laboratory selection during 30 generations to obtain the LiTOX strain. Selection consisted in exposing 6000 third instar larvae to toxic leaf litter to obtain about $70 \%$ of larval mortality after $48 \mathrm{~h}$ exposure [19]. Both susceptible and resistant strains were reared in standard insectary conditions $\left(27^{\circ} \mathrm{C}, 14 / 10 \mathrm{~h}\right.$ light/ dark period, $80 \%$ relative humidity). Larvae were reared in tap water and fed with standard amount of larval food $[19,73]$.

\section{Production of individual Bti Cry toxins}

To produce Bti Cry toxins separately, we used a crystal negative strain of Bacillus thuringiensis var. israelensis (4Q2-81) transformed with the plasmids pHT606, pHT618 or pWF53 producing respectively Cry4A, Cry4B and Cry11 toxins obtained from the Pasteur Institute (Paris, France) or from Prof. B. Federici (University of Riverside, USA). Transformed Bti bacteria were grown on Nutrient Agar solid medium (Sigma Aldrich) supplemented with erythromycin antibiotic $(25 \mu \mathrm{g} / \mathrm{mL})$. Spores and crystals were recovered using cell scrapers (BD Falcon) after 7 days at $30^{\circ} \mathrm{C}$ and purified as previously described [19]. This protocol ensures producing large amount of high quality toxin. Toxins were corun on SDS-PAGE with BSA at five concentrations (from 20, $40,60,80,100 \mu \mathrm{g} / \mathrm{mL}$ ). Intensity of each band was estimated and toxin concentration was calculated using BSA as standard using Imagej software v.1.41o [74].

\section{Bioassays}

Comparative bioassays between the LiTOX and the susceptible Bora-Bora strains were conducted after 30 generations of laboratory selection. Larvae from each strain were exposed to 6 concentrations of Cry4Aa, Cry4Ba, Cry11Aa and commercial Bti (Vectobac WG, 3500 ITU/ $\mathrm{mg}$ ) for $24 \mathrm{~h}$ to obtain $5 \%$ to $95 \%$ mortality. Bioassays were performed in triplicate on 20 third-instar larvae in $50 \mathrm{~mL}$ of insecticide solution or tap water (control) according to the standard bioassay procedure described by the World Health Organisation [75]. LC 50 (lethal concentration for $50 \%$ individuals) were calculated for each strain and each toxin using a probit statistical model with the module 'dose' of XLSTAT v.2009.4.06 (Addinsoft). For each toxin, resistance ratios $\left(R_{50}\right)$ were calculated by dividing $\mathrm{LC}_{50}$ of the LiTOX strain by $\mathrm{LC}_{50}$ of the susceptible strain.

\section{Larval midgut RNA extraction}

For each strain, three biological replicates of 150 dissected midguts from early fourth instar larvae were prepared and conserved overnight at $4^{\circ} \mathrm{C}$ in RNAlater $^{\circledR}$ (Ambion). After a brief centrifugation, supernatant was discarded and total RNA was extracted using RNAqueous ${ }^{\circledR}-4 \mathrm{PCR}$ kit (Ambion) following manufacturer's instructions. Quantity and quality of RNA were assessed by spectrophotometry (Nanodrop ND-1000 spectrophotometer). To digest remaining genomic DNA, RNA samples were treated with DNAseI (Ambion) following manufacturer's instructions. RNA were then concentrated using ammonium acetate and linear acrylamide to obtain at least $70 \mathrm{ng} / \mu \mathrm{L}$ 
of total RNA for each sample. Because the LiTOX strain was selected from the Bora-Bora strain, they share the same genetic background, and both were bred together in the same insectarium standard conditions, so that any constitutive change in gene expression between these strains is likely to result from Bti selection.

\section{Larval midguts transcriptome profiling by DNA microarray}

Low Input Quick Amp Labeling Kit, two-color (Agilent), containing Cy5 and Cy3 fluorescent dyes, was used to amplify and label messenger RNA. Labeled RNAs were then purified using Absolutely RNA ${ }^{\circledR}$ Nanoprep Kit (Stratagene) following manufacturer's instructions with two elution steps in a final volume of $25 \mu \mathrm{L}$. Quantity and quality of RNA and labeling efficiency were assessed using Nanodrop spectrophotometer and Bioanalyzer ${ }^{\circledR}$ (Agilent).

Microarray hybridizations were performed with the $15 \mathrm{~K}$ Agilent 'Aedes detox chip plus' DNA microarray (ArrayExpress accession number A-MEXP-1966), containing eight replicated arrays of 60-mers oligoprobes representing 14204 different Ae. aegypti transcripts and several control probes. For each biological replicate, two hybridizations were performed in which the Cy3 and Cy5 labels were swapped between samples for a total of six hybridizations. For each hybridization, $300 \mathrm{ng}$ of labeled mRNA were used. After $17 \mathrm{~h}$ hybridization, non-specific probes were washed off according to manufacturer's instructions. Slides were scanned with an Agilent G2205B microarray scanner. Spot finding and signal quantification were performed using the Agilent Feature Extraction software (Agilent Technologies).

Data were analyzed using GeneSpring GX v9.0 software (Agilent). Only transcripts present in at least 5 hybridizations out of 6 were kept for further analyses. Transcripts exhibiting more than 3-fold transcription and a Benjamini-Hochberg [76] corrected P-value $<0.01$ were considered significantly differentially transcribed between the LiTOX and the susceptible strain. Midgut transcripts detected by microarrays were then classified into thirteen different categories based on their putative biological functions: receptors, transport, DNA interaction, cytoskeleton, ribosomal proteins, proteases, detoxification enzymes, kinases-phosphatases, transaminases, dehydrogenases, other enzymes, other function and unknown function. For genes of 'unknown function', the putative function was further investigated using BLASTP software, but they were not considered for functional analysis.

\section{Real-time quantitative PCR (RT-qPCR) validation of microarray data}

Transcription levels of 15 genes detected differentially transcribed with the microarray approach were validated by RT-qPCR using the same RNA extracts used in microarrays. In addition, transcription levels of two more genes (ALP1 and Cad1) encoding known Bti Cry toxins binding proteins were also compared between both strains by RTqPCR. Three technical replicates were performed for each of the three biological replicates. Specific primers were designed for each gene using Beacon Designer v.5.10 software (Premier Biosoft International) (Additional file 8). Their specificity to the target gene was verified by BLAST analysis against Ae. aegypti genome. First-strand cDNA synthesis was obtained from $4 \mu \mathrm{g}$ RNA by incubating them at $50^{\circ} \mathrm{C}$ for $1 \mathrm{~h}$ with SuperScript III (Invitrogen) reverse transcriptase, oligo- $\mathrm{dT}_{20}$ primers $(2.5 \mu \mathrm{M})$, dNTPs $(0.5 \mathrm{mM}$ each), DTT (5 mM) and RNase Out (40 U, Invitrogen). Real-time quantitative PCR reaction was performed in $25 \mu \mathrm{L}$ total reaction volume with specific primers $(0.3 \mu \mathrm{M}$ each), $12.5 \mu \mathrm{L}$ iQ SYBR Green supermix (Bio-Rad) and 5 $\mu \mathrm{L}$ diluted cDNA on an iQ5 system (Bio-Rad). After an initial denaturing step at $95^{\circ} \mathrm{C}$ for $3 \mathrm{~min}, 40$ cycles were performed each consisting in a denaturing step $15 \mathrm{~s}$ at $95^{\circ} \mathrm{C}$ and an annealing step $30 \mathrm{~s}$ at the optimal temperature of each primers couple (Additional file 8) [22,71]. Specificity of DNA amplification was assessed by performing a melt curve analysis and verifying PCR product Tm. To check for any contamination, "no template controls" (NTC) were added in each PCR plate.

For each gene analyzed, a serial dilution of pooled cDNA from both strains was used to estimate PCR efficiency. Genes encoding ribosomal proteins RPL8 and RPS7 (housekeeping genes) were used for gene expression normalization taking into account PCR efficiency using $\Delta \Delta \mathrm{C}_{\mathrm{T}}$ method, calculated using the iQ5 software (Bio-Rad) [77,78]. Mean transcription ratios are expressed for the resistant strain relative to the susceptible strain.

\section{GPI-anchor domain detection}

To see whether some midgut enzymes detected with DNA microarrays were membrane-bound, we looked for glycosylphosphatidylinositol (GPI)-anchor domains using four complementary GPI domains predictors: big-PI Predictor v.3.0 (http://mendel.imp.ac.at/gpi/ gpi_server.html) [79], PredGPI (http://gpcr.biocomp. unibo.it/predgpi/pred.htm) [80], FragAnchor (http:// navet.ics.hawaii.edu/ fraganchor/NNHMM/NNHMM. html) [81] and GPI-SOM (http://gpi.unibe.ch/) [82].

\section{Brush border membrane vesicles (BBMV) preparation}

For each strain, two independent biological replicates were prepared. The day before midgut dissection, water 
was changed and food discarded. Early fourth instar larvae were chilled on ice for at least $20 \mathrm{~min}$. Larvae were then dried on a clean paper. Midguts were dissected and mixed together in MET buffer (300 mM Mannitol, 5 mM EGTA, 17 mM TrisHCl, pH 7.5) with Complete Protease Inhibitor (Roche) to be conserved at $-80^{\circ} \mathrm{C}$ until use. About 1500 larvae were dissected for each larval strain and biological replicate. $500 \mu \mathrm{g}$ of midguts were centrifuged $5 \mathrm{~min}$ at $12,000 \mathrm{~g}$ to discard the old buffer, resuspended in ice-cold fresh MET buffer containing $1 \mathrm{mM}$ PMSF and homogenized with 30 strokes of a glass-teflon homogenizer. BBMV were prepared following magnesium precipitation method as previously described [83]. BBMV protein concentration was determined by a Bradford assay using BSA as standard [84]. About 600 dissected guts yielded $500 \mu \mathrm{g}$ of BBMV based on protein amount. Quality of BBMV was assessed by measuring the enrichment of two brush border enzymes: alkaline phosphatases (ALP) and aminopeptidases (APN). ALP and APN activities were measured using 4nitrophenyl phosphate disodium and L-leucine-p-nitroanilide as substrates, respectively $[85,86]$. APN and ALP enrichments are obtained by dividing the activity in the final BBMV preparation by the activity in the initial midgut homogenate (Table 5).

\section{D-DIGE}

$150 \mu \mathrm{g}$ of BBMV proteins from each strain were used for each 2D-DIGE experiment and were purified using 2D-clean up kit (Amersham Bioscience) as described by the manufacturer. $100 \mu \mathrm{g}$ of proteins were labeled with either $\mathrm{Cy} 3$ or $\mathrm{Cy} 5$ and the remaining $50 \mu \mathrm{g}$ of proteins from the each strain were pooled and labeled with $\mathrm{Cy} 2$ as an internal standard. A dye swap was performed to be sure that the observed differences between the two strains were not due to different efficiencies of the dyes to label different proteins. The CyDye minimal labeling of the purified proteins was performed following manufacturer's instructions (GE Healthcare). Labeled proteins were then mixed together and diluted to a final volume of $340 \mu \mathrm{L}$ with rehydration buffer $(2 \mathrm{M}$ Thiourea, $7 \mathrm{M}$ Urea, 3\% CHAPS, 1\% SB3-10, 13 mM DTT, 1\% Immobilized $\mathrm{pH}$ Gradient (IPG) buffer $\mathrm{pH}$ 4-7, 0.002\%

Table 5 APN and ALP enrichments in final BBMV preparation relative to the initial midgut homogenate

\begin{tabular}{llll}
\hline Strain & Biological Replicate & APN enrichment & ALP enrichment \\
\hline Susceptible & First & 4.4 fold & 6.1 fold \\
& Second & 5.3 fold & 1.6 fold \\
\multirow{2}{*}{ LiTOX } & First & 5.0 fold & 7.1 fold \\
& Second & 4.8 fold & 3.2 fold
\end{tabular}

Enrichments are given for each of the two biological replicates for each strain.
Bromophenol blue (w/v)) and loaded on an IPG strip (pH 4-7 nonlinear, $18 \mathrm{~cm}$ ) overlaid with $2 \mathrm{~mL}$ of plusone IPG strip cover fluid (GE Healthcare). After $17 \mathrm{~h}$ of passive rehydration, the first dimension was run on a Multiphor-II flatbed system (GE Healthcare) at $20^{\circ} \mathrm{C}$ with the following program: $15 \mathrm{~min}$ at $300 \mathrm{~V}, 15 \mathrm{~min}$ at $500 \mathrm{~V}$, and $9 \mathrm{~h}$ at $3500 \mathrm{~V}$. This step allows proteins to migrate on the strip till a region in which $\mathrm{pH}$ is equal to their pI (isoelectric point).

After the Isoelectric Focusing, strips were reduced in equilibration buffer (6 M Urea, $75 \mathrm{mM}$ Tris $\mathrm{pH} 8.8,2 \%$ SDS, 29.3\% Glycerol (v/v), 0.002\% Bromophenol blue $(\mathrm{w} / \mathrm{v}))$ containing $1 \%$ of DTT (w/v) for $15 \mathrm{~min}$ and then alkylated in equilibration buffer with $2.5 \%$ of Iodoacetamide for $15 \mathrm{~min}$. The IPG strip was then transferred on a pre-casted $12.5 \%$ SDS-PAGE gel (GE Healthcare) and second dimensional electrophoresis, separating proteins in function of their molecular size, was run at $22^{\circ} \mathrm{C}$ for $1 \mathrm{~h}$ at $2.5 \mathrm{~W} /$ gel followed by $5 \mathrm{~h}$ at $17 \mathrm{~W} /$ gel on an Ettan DALTsix vertical electrophoresis system (GE Healthcare). DIGE Gels were scanned using a Typhoon 9400 imager (GE Healthcare). As CyDye labeling induces a size modification of $1-2 \%$ of the amount of all the proteins that could bias the protein identification, nonlabeled proteins were also prepared in parallel following the same protocol (except CyDye labeling) for mass spectra analyses and regular gels were co-run with DIGE gels to avoid modification in spot patterns due to different migrations. Regular gels were stained with Deep Purple stain (GE Healthcare), scanned using 532/610 nm excitation/emission wavelengths and used for spot picking.

\section{Protein identification}

2D-DIGE gels were analyzed using Decyder v7.0 software (GE Healthcare). The Decyder detection algorithm 5.0 was used to generate a list of spots with their coordinates and level of expression in the resistant strain relative to the susceptible strain. Only spots showing at least 1.5 fold differences between the two strains were considered for further analyses. 29 spots were picked from the first biological replicate and 35 from the second one, with 8 spots shared between them. Excised spots were digested with trypsin before subjecting peptides fragments to MALDI-ToF/ToF (time-of-flight) [29]. To increase the likelihood of protein identification, each protein was identified by searching MS/MS data against an Ae. aegypti local database or other dipteran database when no significant match was obtained. To ensure accurate protein identification, we compared observed and expected pI values, molecular size, percentage of amino acid coverage and Mascot scores for Mascot search engine (http://www. 
matrixscience.com/search_intro.html) or z-scores for ProFound (http://prowl.rockefeller.edu).

\section{Cadherin detection by immunoblotting}

$20 \mu \mathrm{g}$ of proteins from BBMV prepared from the susceptible Bora-Bora strain, the LiTOX strain and the UGAL strain were separated by SDS-PAGE on $4-20 \%$ gradient TGX gels (Biorad). BBMV from the UGAL strain were prepared a few days before the experiments to compare the cadherin conservation in those fresh BBMV to the previously prepared BBMV from the two other strains. Proteins were either stained with coomassie blue to control that equal amount of proteins were stained from all the strains, or electroblotted to polyvinylidene fluoride (PVDF) filters for immunoblotting. Filters were blocked with $3 \%$ bovine serum albumin (BSA) in PBST (PBS + 0.1\% Tween20) for $1 \mathrm{~h}$ at room temperature and then probed with $\alpha$ AgCad1 antibodies (1:5000 dilution) [87], $\alpha$-AgCad2 antibodies (1:500 dilution) or with pre-immune serum from the AgCad2 rabbit in PBST-0.1\% BSA for $2 \mathrm{~h}$. $\alpha-\mathrm{AgCad} 2$ antibodies were prepared against an E. coli expressed cadherin peptide AgCad2 (Hua et al., unpublished work). Filters were then washed and detected by an anti-rabbit IgG-peroxidase conjugate (1:25000 dilution) in PBST-0.1\% BSA for $1 \mathrm{~h}$ at room temperature. Filters were developed with an ECL kit (GE Healthcare) and chemiluminescence was detected with a ChemiImager (Alpha Innotech). All the Western blots were performed in duplicate.

\section{Larval midgut proteolytic activities}

For each strain, three biological replicates of soluble protein extracted from midgut juice were prepared. 20 midguts of early fourth instars were extracted and placed into $50 \mu \mathrm{L}$ of distilled water and homogenized using a vortex for $30 \mathrm{~s}$. Sample were centrifuged at 12,000 g for $10 \mathrm{~min}$ at $4{ }^{\circ} \mathrm{C}$. All the supernatants from larvae of the same biological replicate were mixed together, protein concentration was quantified by a Bradford assay using BSA as standard [84] and aliquots were conserved at $-20^{\circ} \mathrm{C}$ until use. Total protease activity was measured using azocasein as substrate (Sigma Aldrich) as described in [88]. All activities were normalized according to the amount of total protein from each replicate. For each biological replicate, six technical replicates were performed and absorbance was measured at $440 \mathrm{~nm}$. Percentages of protease activity due to serine proteases, chymotrypsins, trypsins and metallo-enzymes were measured using respectively PMSF $(30 \mathrm{mM})$, TPCK $(1.5 \mathrm{mM})$, TLCK $(1.5 \mathrm{mM})$ and EDTA (1 mM) (Sigma Aldrich) [88]. Statistical differences between the two strains were measured by a Wilcoxon test performed with R 2.8.1 software [89].

\section{Additional files}

Additional file 1: All the $\mathbf{3 5 1 2}$ transcripts detected by microarrays experiments in at least $\mathbf{5}$ hybridizations out of $\mathbf{6}$. For each transcript, accession number, corrected p-value, expression level changes, Vectorbase annotation and functional category are indicated.

Additional file 2: 70 transcripts significantly (corrected $\mathrm{P}$-val $<0.01$ ) more than 3-fold differentially transcribed in the LiTOX strain.

Transcripts are classified according to their putative function using the 13 functional categories. For each transcript, accession number, corrected Pvalue, expression level changes, Vectorbase annotation and supercontig are indicated. For transcripts of 'unknown functions', their putative function with corresponding score, ID, accession number and species of the best hit found using BLASTP software are indicated.

Additional file 3: Validation of microarray data by RT-qPCR on fifteen selected genes. Both experiments were performed on the same mRNA extracted from dissected larval midguts. ALP2, Alkaline phosphatase AAEL003298; ALP3, AAEL003313; ALP5, AAEL015070; ALP6, AAEL011175; APN1, N-Aminopeptidase AAEL012774; APN2, AAEL012776; APN3, AAEL012778; Cad2, Cadherin AAEL007488; HP1, Conserved hypothetical protein AAEL010435; HP2, AAEL013584; SE1, Serine-type endopeptidase AAEL007938; SE2, Serine-type endopeptidase AAEL011917; Cytochrome P450: CYP6Z7, AAEL009130; CYP6Z8, AAEL009131 and CYP4D24, AAEL007815.

Additional file 4: 2D-DIGE gels from the two biological replicates and dye-swapping. BBMV prepared from first ( $A$ and $B$ ) and second ( $C$ and $\mathrm{D})$ biological replicate are separated in function of their size $(\mathrm{kDa})$ and their isoelectric point (pl). BBMV from Bti resistant strain are labeled with Cy3 and susceptible strain with Cy5 (A and C) or resistant strain with Cy5 and susceptible with Cy3 (B and D).

Additional file 5: Protein identification of the $\mathbf{5 6}$ spots picked on deep purple stained 2D-gel. When different spots pointed to the same protein, they were differentiated using arbitrary letters after the access number. For each identification, the predicted pl, the predicted mass in kilodaltons, the percentage of sequence coverage, their functional category, and the species and database matched are indicated.

Additional file 6: Glycosylphosphatidylinositol (GPI)-anchor domains detection by four predictive computational programs. For each gene and protein, their accession number, the transcript and protein sizes are indicated. Results from the big-GPI and GPI-SOM softwares are indicated as 'YES' when they found a potential GPI-domain and 'NO' when no GPI-domain was determined. For PredGPI, presence is indicated by 'Highly probable', 'Weakly probable' or 'Probable' and absence by 'NO'. For FragAnchor, presence of GPI domain is indicated by 'Highly probable' or 'Probable', absence by 'NO' and when prediction is uncertain by 'Potential false positive'

Additional file 7: Cadherin detection by immunoblotting. BBMV proteins from the susceptible Bora-Bora strain (lane 1), LiTOX strain (lane 2) and the UGAL Aedes strain (lane 3) were separated in SDS-PAGE and stained with coomassie blue (panel A) or probed with a-AgCad1 antibodies (panel B), a-AgCad2 antibodies (panel C) or with pre-immune serum from a-AgCad2 rabbit (panel D).

Additional file 8: Primer pairs used for RT-qPCR analyses. For each primer pair, sequence, corresponding gene name and accession number, product length, Tm and optimal annealing temperature used in PCR program are indicated. PCR efficiency and different parameters of the calibration curves ( $R^{2}$, slope and $y$-intercept) are also indicated. Specificity of each primer pair was first assessed by BLAST analysis against Ae. aegypti genome and then verified by performing a melt curve analysis. A high specificity is indicated as "YES" when the primer pair matched to a unique position in the Ae. aegypti genome and when PCR product Tm was correct.

\section{Abbreviations}

ALP: Alkaline phosphatase; APN: N-aminopeptidase; BBMV: Brush border membrane vesicles; DIGE: Differential in gel electrophoresis;

EDTA: Ethylenediaminetetraacetic acid; GPI: Glycosylphosphatidylinositol; MS/ MS: Tandem mass spectrometry; PMSF: Phenylmethylsulfonyl fluoride; 
PVDF: Polyvinylidene fluoride; TLCK: $\mathrm{N}_{a}$-tosyl-L-lysine chloromethyl ketone hydrochloride; TPCK: N-p-Tosyl-L-phenylalanine chloromethyl ketone.

\section{Competing interests}

The authors declare that no competing interests exist.

\section{Author's contributions}

J. P.D., M.J.A. and L.D. designed research; G.T. and K.B. performed DiGE experiments; K.B. did Western blot experiments; G.T., K.B. and M.J.A. analyzed proteomic data; G.T. and M.A.R. prepared mRNA samples for microarrays and did gene expression analyses; C.M.J. performed microarray experiment; G.T. performed RT-qPCR experiments; G.T. and J.P.D analyzed transcriptomic data; G.T. and R.S. did enzymatic experiments; G.T. and M.P. performed the selection and rearing of the LiTOX strain; G.T. wrote the paper; K.B., R.S., J.P.D., M.J.A. and L.D. reviewed and helped improving the manuscript. All authors read and approved the manuscript.

\section{Author's information}

G.T. performed this study during his Ph.D. at the Laboratoire d'Ecologie Alpine (LECA), University of Grenoble. His research interests goes from the understanding of the fate of pesticides in the field to the adaptive strategies of target insects. K.B. worked on this study during his Ph.D. tenure in the Dept. of Entomology, University of Georgia. Currently, he is a Postdoctoral Research Associate in the Dept. of Microbiology, UGA. His research interest is Oxidative protein damage and protein repair in Helicobacter pylori. C.M.J. is a post-doctoral researcher in the Vector Group of the LSTM where his current interests lie in vector biology and genetics with a strong emphasis on insecticide resistance. R.S. participated to this study during his Master internship at the LECA. He is interested in molecular ecology and agricultural science. M.P. participated to this study during her Ph.D. She is now a postdoc in Zurich, and is interested in population genetics, molecular adaptation and evolution. J.P.D. is a senior CNRS researcher having a strong experience in vector control and adaptive mechanisms developed by insects to insecticides, pollutants and toxins. M.J.A., a professor at the University of Georgia and CSO of InsectiGen, investigates Bt toxins and their action in pest insects. L.D. is a Professor at University of Grenoble. Her main research interests are in adaptive patterns in natural populations and underlying evolutionary processes.

\section{Acknowledgments}

The authors would like to thank B. Federici for providing recombinant $B t$ strains. They also thank E. Bianco, A. Bonin and A. Proust for their help for larval midgut dissections. They thank S. Veyrenc and T. Gaude for technical assistance with mosquito breeding and insectary maintenance. They thank Dr. B. MacCallum for useful help with microarray data deposition in Vectorbase. This study was founded by the French National Research Agency (ANR, project ANR-08-CES-006-01 DIBBECO). Guillaume Tetreau was supported by the French Ministry of Research.

\section{Author details}

'Laboratoire d'Ecologie Alpine, LECA-UMR 5553, Université de Grenoble 1, BP 53, 38041, Grenoble cedex 09, France. ${ }^{2}$ Department of Entomology, University of Georgia, Athens, GA 30602-2603, USA. ${ }^{3}$ Vector Group, Liverpool School of Tropical Medicine, Liverpool L3 5QA, UK. ${ }^{4}$ Department of Biochemistry and Molecular Biology, University of Georgia, Athens, GA 30602-2603, USA.

Received: 13 March 2012 Accepted: 25 May 2012

Published: 15 June 2012

\section{References}

1. Hemingway J, Ranson H: Insecticide resistance in insect vectors of human disease. Annu Rev Entomol 2000, 45:371-391.

2. Thai KTD, Anders KL: The role of climate variability and change in the transmission dynamics and geographic distribution of dengue. Exp Biol Med 2011, 236(8):944-954.

3. Weaver SC, Reisen WK: Present and future arboviral threats. Antivir Res 2010, 85(2):328-345.

4. Kyle JL, Harris E: Global spread and persistence of dengue. Annu Rev Microbiol 2008, 62:71-92.
5. Tomori O: Yellow fever: the recurring plague. Crit Rev Clin Lab Sci 2004, 41(4):391-427

6. van den Berg $\mathrm{H}$ : Global status of DDT and its alternatives for use in vector control to prevent disease. Cienc Saude Coletiva 2011, 16(2):575590.

7. Lacey LA: Bacillus thuringiensis serovariety israelensis and Bacillus sphaericus for mosquito control. J Am Mosq Control Assoc 2007, 23(2):133163.

8. Bravo A, Gill SS, Soberon M: Mode of action of Bacillus thuringiensis Cry and Cyt toxins and their potential for insect control. Toxicon 2007, 49(4):423-435.

9. Bravo A, Likitvivatanavong S, Gill SS, Soberon M: Bacillus thuringiensis: a story of a successful bioinsecticide. Insect Biochem Mol Biol 2011, 41(7):423-431.

10. Gassmann AJ, Petzold-Maxwell JL, Keweshan RS, Dunbar MW: Field-evolved resistance to Bt maize by Western corn rootworm. PLoS One 2011, 6(7): e22629.

11. Dhurua S, Gujar GT: Field-evolved resistance to $B t$ toxin Cry1Ac in the pink bollworm, Pectinophora gossypiella (Saunders) (Lepidoptera: Gelechiidae), from India. Pest Manag Sci 2011, 67(8):898-903.

12. Tabashnik BE, Van Rensburg JBJ, Carriere Y: Field-evolved insect resistance to Bt crops: definition, theory, and data. J Econ Entomol 2009, 102(6):2011-2025.

13. Storer NP, Babcock JM, Schlenz M, Meade T, Thompson GD, Bing JW, Huckaba RM: Discovery and characterization of field resistance to $B t$ maize: Spodoptera frugiperda (Lepidoptera: Noctuidae) in Puerto Rico. $J$ Econ Entomol 2010, 103(4):1031-1038.

14. Paul A, Harrington LC, Zhang L, Scott JG: Insecticide resistance in Culex pipiens from New York. J Am Mosa Control Assoc 2005, 21(3):305-309.

15. Wirth MC, Park HW, Walton WE, Federici BA: Cyt1A of Bacillus thuringiensis delays evolution of resistance to Cry $11 \mathrm{~A}$ in the mosquito Culex quinquefasciatus. Appl Environ Microbiol 2005, 71(1):185-189.

16. Tilquin M, Paris M, Reynaud S, Despres L, Ravanel P, Geremia RA, Gury J: Long lasting persistence of Bacillus thuringiensis Subsp israelensis (Bti) in mosquito natural habitats. PLoS One 2008, 3(10):e3432.

17. de Melo-Santos MAV, de Araujo AP, Rios EMM, Regis L: Long lasting persistence of Bacillus thuringiensis serovar. israelensis larvicidal activity in Aedes aegypti (Diptera: Culicidae) breeding places is associated to bacteria recycling. Biol Control 2009, 49(2):186-191.

18. Shaheen R, Svensson B, Andersson MA, Christiansson A, Salkinoja-Salonen M: Persistence strategies of Bacillus cereus spores isolated from dairy silo tanks. Food Microbiol 2010, 27(3):347-355.

19. Paris M, Tetreau G, Laurent F, Lelu M, Despres L, David J-P: Persistence of Bacillus thuringiensis israelensis (Bti) in the environment induces resistance to multiple Bti toxins in mosquitoes. Pest Manag Sci 2011, 67:122-128.

20. Goldman IF, Arnold J, Carlton BC: Selection for resistance to Bacillus thuringiensis subspecies israelensis in field and laboratory populations of the mosquito Aedes aegypti. J Invertebr Pathol 1986, 47(3):317-324.

21. Mittal PK, Adak T, Subbarao SK: Laboratory selection to investigate the development of resistance to Bacillus thuringiensis var. israelensis $\mathrm{H}-14$ in Culex quinquefasciatus Say (Diptera: Culicidae). Natl Acad Sci Lett-India 2005, 28(7-8):281-283.

22. Bonin A, Paris M, Tetreau G, David JP, Despres L: Candidate genes revealed by a genome scan for mosquito resistance to a bacterial insecticide: sequence and gene expression variations. BMC Genomics 2009, 10:551.

23. Paris M, Melodelima C, Coissac E, Tetreau G, Reynaud S, David JP, Despres L: Transcription profiling of resistance to $B t i$ toxins in the mosquito Aedes aegypti using next-generation sequencing. I Invertebr Pathol 2012, 109 (2):201-208

24. Paris $M, D a v i d J P$, Despres $L$ : Fitness costs of resistance to Bti toxins in the dengue vector Aedes aegypti. Ecotoxicology 2011, 20:1184-1194.

25. Georghiou GP, Wirth MC: Influence of exposure to single versus multiple toxins of Bacillus thuringiensis subsp. israelensis on development of resistance in the mosquito Culex quinquefasciatus (Diptera: Culicidae). Appl Environ Microbiol 1997, 63(3):1095-1101.

26. Saleh MS, El-Meniawi FA, Kelada NL, Zahran HM: Resistance development in mosquito larvae Culex pipiens to the bacterial agent Bacillus thuringiensis var. israelensis. J Appl Entomol-Zeitschrift Fur Angew Entomol 2003, 127(1):29-32. 
27. Griffitts JS, Aroian RV: Many roads to resistance: how invertebrates adapt to Bt toxins. BioEssays 2005, 27(6):614-624.

28. Popova-Butler A, Dean DH: Proteomic analysis of the mosquito Aedes aegypti midgut brush border membrane vesicles. J Insect Physiol 2009, 55(3):264-272

29. Bayyareddy K, Andacht TM, Abdullah MA, Adang MJ: Proteomic identification of Bacillus thuringiensis subsp israelensis toxin Cry4Ba binding proteins in midgut membranes from Aedes (Stegomyia) aegypti Linnaeus (Diptera, Culicidae) larvae. Insect Biochem Mol Biol 2009, 39(4):279-286

30. Gygi SP, Rochon Y, Franza BR, Aebersold R: Correlation between protein and mRNA abundance in yeast. Mol Cell Biol 1999, 19(3):1720-1730

31. De Wit M, Keil D, van der Ven K, Vandamme S, Witters E, De Coen W: An integrated transcriptomic and proteomic approach characterizing estrogenic and metabolic effects of 17 alpha-ethinylestradiol in zebrafish (Danio rerio). Gen Comp Endocrinol 2010, 167(2):190-201.

32. Foss EJ, Radulovic D, Shaffer SA, Goodlett DR, Kruglyak L, Bedalov A Genetic variation shapes protein networks mainly through nontranscriptional mechanisms. PLoS Biol 2011, 9(9):e1001144.

33. Likitvivatanavong S, Chen JW, Evans AM, Bravo A, Soberon M, Gill SS: Multiple receptors as targets of cry toxins in mosquitoes. $J$ Agric Food Chem 2011, 59(7):2829-2838.

34. Chen JW, Aimanova KG, Fernandez LE, Bravo A, Soberon M, Gill SS: Aedes aegypti cadherin serves as a putative receptor of the Cry11Aa toxin from Bacillus thuringiensis subsp israelensis. Biochem J 2009, 424:191-200.

35. Chen JW, Aimanova KG, Pan SQ, Gill SS: Identification and characterization of Aedes aegypti aminopeptidase $\mathrm{N}$ as a putative receptor of Bacillus thuringiensis Cry11A toxin. Insect Biochem Mol Biol 2009, 39(10):688-696.

36. Fernandez LE, Aimanova KG, Gill SS, Bravo A, Soberon M: A GPI-anchored alkaline phosphatase is a functional midgut receptor of Cry11Aa toxin in Aedes aegypti larvae. Biochem J 2006, 394:77-84.

37. McNall RJ, Adang MJ: Identification of novel Bacillus thuringiensis Cry1AC binding proteins in Manduca sexta midgut through proteomic analysis. Insect Biochem Mol Biol 2003, 33(10):999-1010.

38. Arenas I, Bravo A, Soberon M, Gomez I: Role of alkaline phosphatase from Manduca sexta in the mechanism of action of Bacillus thuringiensis Cry1Ab toxin. J Biol Chem 2010, 285(17):12497-12503.

39. Martins ES, Monnerat RG, Queiroz PR, Dumas VF, Braz SV, Aguiar RWD, Gomes A, Sanchez J, Bravo A, Ribeiro BM: Midgut GPI-anchored proteins with alkaline phosphatase activity from the cotton boll weevil (Anthonomus grandis) are putative receptors for the Cry1B protein of Bacillus thuringiensis. Insect Biochem Mol Biol 2010, 40(2):138-145.

40. Hua G, Zhang R, Bayyareddy K, Adang MJ: Anopheles gambiae alkaline phosphatase is a functional receptor of Bacillus thuringiensis jegathesan Cry11Ba toxin. Biochemistry 2009, 48(41):9785-9793.

41. Jurat-Fuentes JL, Karumbaiah L, Jakka SRK, Ning CM, Liu CX, Wu KM, Jackson J, Gould F, Blanco C, Portilla M, et al: Reduced levels of membrane-bound alkaline phosphatase are common to Lepidopteran strains resistant to cry toxins from Bacillus thuringiensis. PLoS One 2011, 6(3):e17606.

42. Pigott CR, Ellar DJ: Role of receptors in Bacillus thuringiensis crystal toxin activity. Microbiol Mol Biol Rev 2007, 71(2):255.

43. Abdullah MAF, Moussa S, Taylor MD, Adang MJ: Manduca sexta (Lepidoptera: Sphingidae) cadherin fragments function as synergists for Cry $1 \mathrm{~A}$ and $\mathrm{Cry} 1 \mathrm{C}$ Bacillus thuringiensis toxins against noctuid moths Helicoverpa zea, Agrotis ipsilon and Spodoptera exigua. Pest Manag SCi 2009, 65(10):1097-1103.

44. Saengwiman S, Aroonkesorn A, Dedvisitsakul P, Sakdee S, Leetachewa S, Angsuthanasombat C, Pootanakit K: In vivo identification of Bacillus thuringiensis Cry4Ba toxin receptors by RNA interference knockdown of glycosylphosphatidylinositol-linked aminopeptidase $\mathrm{N}$ transcripts in Aedes aegypti larvae. Biochem Biophys Res Commun 2011, 407(4):708-713.

45. Zhang SP, Cheng HM, Gao YL, Wang GR, Liang GM, Wu KM: Mutation of an aminopeptidase $\mathrm{N}$ gene is associated with Helicoverpa armigera resistance to Bacillus thuringiensis Cry1Ac toxin. Insect Biochem Mol Biol 2009, 39(7):421-429.

46. Tiewsiri K, Wang P: Differential alteration of two aminopeptidases $\mathrm{N}$ associated with resistance to Bacillus thuringiensis toxin Cry1 Ac in cabbage looper. Proc Natl Acad Sci U S A 2011, 108(34):14037-14042.
47. Ferre J, Escriche B, Bel Y, Vanrie J: Biochemistry and genetics of insect resistance to Bacillus-thuringiensis insecticidal crystal proteins. FEMS Microbiol Lett 1995, 132(1-2):1-7.

48. Yang YL, Zhu YC, Ottea J, Husseneder C, Leonard BR, Abel C, Luttrell R, Huang FN: Down regulation of a gene for cadherin, but not alkaline phosphatase, associated with Cry1 Ab resistance in the sugarcane borer Diatraea saccharalis. PLoS One 2011, 6(10):e25783.

49. Herrero S, Gechev T, Bakker PL, Moar WJ, de Maagd RA: Bacillus thuringiensis Cry1Ca-resistant Spodoptera exigua lacks expression of one of four Aminopeptidase N genes. BMC Genomics 2005, 6(96).

50. Jurat-Fuentes $J \mathrm{~L}$, Adang MJ: Characterization of a Cry1Ac-receptor alkaline phosphatase in susceptible and resistant Heliothis virescens larvae. Eur J Biochem 2004, 271(15):3127-3135.

51. Perez C, Fernandez LE, Sun JG, Folch JL, Gill SS, Soberon M, Bravo A: Bacillus thuringiensis subsp israelensis Cyt1Aa synergizes Cry11Aa toxin by functioning as a membrane-bound receptor. Proc Natl Acad Sci U S A 2005, 102(51):18303-18308.

52. Ravoahangimalala O, Charles JF: In-vitro binding of Bacillus-thuringiensis var israelensis individual toxins to midgut cells of Anopheles-gambiae larvae (Diptera, Culicidae). FEBS Lett 1995, 362(2):111-115.

53. Yamagiwa M, Kamauchi S, Okegawa T, Esaki M, Otake K, Amachi T, Komano $\mathrm{T}$, Sakai H: Binding properties of Bacillus thuringiensis Cry4A toxin to the apical microvilli of larval midgut of Culex pipiens. Biosci Biotechnol Biochem 2001, 65(11):2419-2427.

54. Krishnamoorthy M, Jurat-Fuentes JL, McNall RJ, Andacht T, Adang MJ: Identification of novel CrylAc binding proteins in midgut membranes from Heliothis virescens using proteomic analyses. Insect Biochem Mol Biol 2007, 37(3):189-201.

55. Chen LZ, Liang GM, Zhang J, Wu KM, Guo YY, Rector BG: Proteomic analysis of novel Cry1 Ac binding proteins in Helicoverpa armigera (Hubner). Arch Insect Biochem Physiol 2010, 73(2):61-73.

56. Harvey WR, Okech BA, Linser PJ, Becnel JJ, Ahearn GA, Sterling KM: H+ VATPase-energized transporters in brush border membrane vesicles from whole larvae of Aedes aegypti. J Insect Physiol 2010, 56(10):1377-1389.

57. Ravoahangimalala O, Charles JF, Schoellerraccaud J: Immunological localization of Bacillus-thuringiensis serovar israelensis toxins in midgut cells of intoxicated Anopheles-gambiae larvae (Diptera, Culicidae). Res Microbiol 1993, 144(4):271-278.

58. Ruiz LM, Segura C, Trujillo J, Orduz S: In vivo binding of the Cry11Bb toxin of Bacillus thuringiensis subsp medellin to the midgut of mosquito larvae (Diptera: Culicidae). Memorias Do Inst Oswaldo Cruz 2004, 99(1):73-79.

59. Onken H, Moffett DF: Revisiting the cellular mechanisms of strong luminal alkalinization in the anterior midgut of larval mosquitoes. J Exp Biol 2009, 212(3):373-377

60. Boudko DY, Moroz LL, Linser PJ, Trimarchi JR, Smith PJS, Harvey WR: In situ analysis of $\mathrm{pH}$ gradients in mosquito larvae using noninvasive, selfreferencing, $\mathrm{pH}$-sensitive microelectrodes. J Exp Biol 2001, 204(4):691-699.

61. Beyenbach KW, Wieczorek H: The V-type H+ ATPase: molecular structure and function, physiological roles and regulation. J Exp Biol 2006, 209 (4):577-589.

62. Onken $\mathrm{H}$, Moffett SB, Moffett DF: Alkalinization in the isolated and perfused anterior midgut of the larval mosquito, Aedes aegypti. J Insect Sci (Online) 2008, 8:1-20.

63. Feng $\mathrm{Q}$, Becktel WJ: $\mathrm{pH}$-induced conformational transitions of Cry IA(a), Cry IA(c), and Cry IIIA delta-endotoxins in Bacillus-thuringiensis. Biochemistry 1994, 33(28):8521-8526.

64. Venugopal MG, Wolfersberger MG, Wallace BA: Effects of $\mathrm{pH}$ on conformational properties related to the toxicity of Bacillus-thuringiensis delta-endotoxin. Biochim Biophys Acta 1992, 1159(2):185-192

65. Karumbaiah L, Oppert B, Jurat-Fuentes JL, Adang MJ: Analysis of midgut proteinases from Bacillus thuringiensis-susceptible and -resistant Heliothis virescens (Lepidoptera: Noctuidae). Comp Biochem Physiol B-Biochem Mol Biol 2007, 146(1):139-146.

66. Tran $L B$, Vachon $V$, Schwartz $J$, Laprade R: Differential effects of $p H$ on the pore-forming properties of Bacillus thuringiensis insecticidal crystal toxins. Appl Environ Microbiol 2001, 67(10):4488-4494. 
67. Fortier M, Vachon V, Kirouac M, Schwartz JL, Laprade R: Differential effects of ionic strength, divalent cations and $\mathrm{pH}$ on the pore-forming activity of Bacillus thuringiensis insecticidal toxins. J Membr Biol 2005, 208(1):77-87.

68. Despres L, David JP, Gallet C: The evolutionary ecology of insect resistance to plant chemicals. Trends Ecol Evol 2007, 22(6):298-307.

69. Feyereisen R: Insect CYP genes and P450 enzymes. In Insect Molecular Biology and Biochemistry. Edited by Gilbert LI.: Academic Press; 2011:236-316.

70. Zeng RS, Wen Z, Niu G, Schuler MA, Berenbaum MR: Enhanced toxicity and induction of cytochrome P450s suggest a cost of "eavesdropping" in a multitrophic interaction. J Chem Ecol 2009, 35(5):526-532.

71. Poupardin R, Reynaud S, Strode C, Ranson H, Vontas J, David JP: Crossinduction of detoxification genes by environmental xenobiotics and insecticides in the mosquito Aedes aegypti: impact on larval tolerance to chemical insecticides. Insect Biochem Mol Biol 2008, 38(5):540-551.

72. David JP, Boyer S, Mesneau A, Ball A, Ranson H, Dauphin-Villemant C: Involvement of cytochrome P450 monooxygenases in the response of mosquito larvae to dietary plant xenobiotics. Insect Biochem Mol Biol 2006 36(5):410-420

73. Riaz MA, Poupardin R, Reynaud S, Strode C, Ranson H, David JP: Impact of glyphosate and benzo[a]pyrene on the tolerance of mosquito larvae to chemical insecticides. Role of detoxification genes in response to xenobiotics. Aquat Toxicol 2009, 93(1):61-69.

74. Girish V, Vijayalakshmi A: Affordable image analysis using NIH Image/ ImageJ. Indian J Cancer 2004, 41(1):47.

75. WHO: Guidelines for Laboratory and Field Testing of Mosquito Larvicides. Geneva: World Health Organization; 2005. WHO/CDS/WHOPES/GCDPP/ 2005.13.

76. Benjamini $Y$, Hochberg $Y$ : Controlling the false discovery rate-a practical and powerful approach to multiple testing. J R Stat Soc Ser B-Methodo 1995, 57(1):289-300.

77. Pfaffl MW: A new mathematical model for relative quantification in realtime RT-PCR. Nucleic Acids Res 2001, 29(9):e45.

78. Poupardin R, Riaz MA, Vontas J, David JP, Reynaud S: Transcription profiling of eleven cytochrome P450s potentially involved in xenobiotic metabolism in the mosquito Aedes aegypti. Insect Mol Biol 2010, 19(2):185-193.

79. Eisenhaber B, Bork P, Yuan YP, Loffler G, Eisenhaber F: Automated annotation of GPI anchor sites: case study C-elegans. Trends in Biochem Sci 2000, 25(7):340-341.

80. Pierleoni A, Martelli PL, Casadio R: PredGPI: a GPI-anchor predictor. BMC Bioinforma 2008, 9

81. Poisson G, Chauve C, Chen X, Bergeron A: FragAnchor: a large-scale predictor of glycosylphosphatidylinositol anchors in eukaryote protein sequences by qualitative scoring. Genomics, Proteomics bioinforma / Beijing Genomics Inst 2007, 5(2):121-130.

82. Fankhauser N, Maser P: Identification of GPI anchor attachment signals by a Kohonen self-organizing map. Bioinformatics 2005, 21(9):1846-1852.

83. Abdul-Rauf M, Ellar DJ: Isolation and characterization of brush border membrane vesicles from whole Aedes aegypti larvae. J Invertebr Pathol 1999, 73(1):45-51.

84. Bradford MM: Rapid and sensitive method for quantitation of microgram quantities of protein utilizing principle of protein-dye binding. Anal Biochem 1976, 72(1-2):248-254

85. Terra WR, Ferreira C: Insect digestive enzymes-properties, compartmentalization and function. Comp Biochem Physiol B-Biochem Mol Biol 1994, 109(1):1-62.

86. Hernandez-Martinez P, Navarro-Cerrillo G, Caccia S, de Maagd RA, Moar WJ Ferre J, Escriche B, Herrero S: Constitutive activation of the midgut response to Bacillus thuringiensis in Bt-resistant Spodoptera exigua. PLoS One 2010, 5(9):e12795.

87. Hua G, Zhang R, Abdullah MAF, Adang MJ: Anopheles gambiae cadherin $\mathrm{AgCad} 1$ binds the Cry4Ba toxin of Bacillus thuringiensis israelensis and a fragment of AgCad1 synergizes toxicity. Biochemistry 2008 47(18):5101-5110.
88. Hosseininaveh V, Bandani A, Azmayeshfard P, Hosseinkhani S, Kazzazi M: Digestive proteolytic and amylolytic activities in Trogoderma granarium Everts (Dermestidae: Coleoptera). J Stored Prod Res 2007, 43:515-522.

89. R Development Core Team: R: A Language and Environment for Statistical Computing:: 2007. http://www.R-project.org.

doi:10.1186/1471-2164-13-248

Cite this article as: Tetreau et al.: Larval midgut modifications associated with Bti resistance in the yellow fever mosquito using proteomic and transcriptomic approaches. BMC Genomics 2012 13:248.

\section{Submit your next manuscript to BioMed Central and take full advantage of:}

- Convenient online submission

- Thorough peer review

- No space constraints or color figure charges

- Immediate publication on acceptance

- Inclusion in PubMed, CAS, Scopus and Google Scholar

- Research which is freely available for redistribution 